\title{
AN OPERATIONAL APPROACH FOR HYPERSPECTRAL IMAGE COMPRESSION
}

\author{
Qian Du, Nam Ly, James E Fowler \\ Department of Electrical and Computer Engineering, Geosystems Research Institute \\ Mississippi State University, USA
}

\begin{abstract}
In lossy compression such as PCA+JPEG2000 for hyperspectral imagery, the bitrate is usually not fixed, resulting in various rate-distortion performance. In this paper, we propose an operational approach to determine the approximately optimal bitrate to be used to preserve both the majority of the information in the dataset as well as the anomalous pixels. The classification results using the reconstructed data after compression with this bitrate are comparable to those using the original data without compression; meanwhile, detection accuracy can be $100 \%$ if all the anomalies are pre-removed before compression.
\end{abstract}

Index Terms - hyperspectral image, compression, classification, anomaly detection.

\section{INTRODUCTION}

It has been shown that principal component analysis (PCA) in conjunction with JPEG2000 can provide superior rate-distortion performance for hyperspectral image compression. In such PCA+JPEG2000 coding, PCA provides spectral decorrelation while the discrete wavelet transform (DWT) within JPEG2000 provides spatial decorrelation of the principal component (PC) images. In particular, PCA+JPEG2000 outperforms DWT+JPEG2000 wherein a DWT is adopted for spectral decorrelation [1-3]. Consequently, spectral decorrelation can be considered to be crucial for hyperspectral compression, and PCA outperforms the DWT in this respect. When only the most important, or major, PCs are retained for compression (i.e., the minor PCs are explicitly discarded prior to the application of JPEG2000 coding), the resulting algorithm was denoted as SubPCA+JPEG2000 in [4]; SubPCA+JPEG2000 can yield even better performance than PCA+JPEG2000, particularly at low bitrates. For example, Fig. 1 shows rate-distortion performance for the coding of an AVIRIS image; Fig. 1 also includes two other prominent DWT-based algorithms: 3D SPIHT [5] and 3D SPECK [6].
Clearly, the PCA-based group of algorithms significantly outperforms the DWT-based group. For SubPCA+JPEG2000, Fig. 2 indicates that the rate-distortion performance varies with the number of PCs which are retained for compression - it is seen that the best performance (i.e., maximum SNR) does not occur with all the 224 PCs being used. The possible reasons discussed in [4] are: 1) the minor PCs may contain noise only, and it is therefore best to assign zero bits to them (by explicitly discarding them), thereby reserving more bits for the major PCs in the JPEG2000 bit allocation; and 2) each packet in the JPEG2000 bitstream contains a header even if the payload is empty, meaning that minor PCs which should be assigned zero bitrate actually end up consuming nonnegligible bitrate in the final bitstream.

In many applications, anomalies in a hyperspectral image are of paramount importance. In [7], hyperspectral compression with the goal of anomaly preservation was proposed. In [7], anomalies are first detected and then preserved without compression as overhead in the compressed bitstream; the remainder of the image is encoded using a traditional coder (e.g., PCA+JPEG2000 or SubPCA+JPEG2000). Before encoding, anomalous pixels are replaced by interpolation from neighboring pixels; in the decoding step, the original anomalous pixels are replaced back. This approach was referred to as anomaly removal (AR) in [8]; we thus refer to the resulting compression algorithms as AR+PCA+JEPG2000 and $\mathrm{AR}+\mathrm{SubPCA}+\mathrm{JPEG} 2000$. Alternatively, in [8] an anomalyadjusted (AA) approach was proposed, wherein anomalous pixels are compressed after mean removal. As demonstrated in Fig. 3, the SubPCA versions outperform the corresponding PCA versions in terms of rate-distortion performance.

The aforementioned lossy compression techniques provide a certain level of distortion performance for a given birate. The design of the JPEG2000 algorithm ensures that it operates at approximately optimal SNR performance for a given bitrate. However, when SubPCA is used for spectral 
decorrelation, one must also select the number of PCs retained. Intuitively, we want to retain the major PCs only, since they contain the majority of the true information content of the dataset; however, since anomalies tend to reside in minor components, we should extract anomalous pixels before applying SubPCA+JPEG2000. In this paper, we propose an operational approach that determines the optimal bitrate for AR+SubPCA+JPEG2000.

\section{PROPOSED WORK}

A. Estimation of the Number of Signal Subspaces and Anomalous Pixels

Hyperspectral signal subspace identification is important in the analysis of hyperspectral imagery; it is related to the virtual dimensionality or the number of spectrally distinctive signal sources [9-10]. The central idea is identification of the significant PCs of the data covariance matrix. In [11], an approach for signal subspace identification was proposed with the special consideration of anomalous pixels. The basic approach is: 1) apply the NWHFC method from [9] to detect the number significant PCs which is denoted as $N_{s} ; 2$ ) project the original data into the orthogonal signal subspace with the pixel with the largest norm in the orthogonal subspace being considered as an anomaly; 3) project the original data to the subspace orthogonal to the signals as well as the detected anomaly, with the pixel with the largest norm in that subspace being considered as the second anomaly; 4) the process is continued until the maximum norm in the orthogonal subspace is small enough (based on an $F$ test). The number of detected anomalous pixels is denoted as $N_{a}$.

\section{B. The Optimal Number of PCs for SubPCA+JPEG2000}

From Fig. 2, we conclude that the optimal number of PCs for SubPCA+JPEG2000 is related to the encoding bitrate. It is also clearly related to image data itself, such as the number of bands and image content. Although there is no theoretical close-form relationship, an empirical piecewise-linear formula was developed for AVIRIS sensor in [12]:

$$
N_{P C}=f(b)=\left\{\begin{array}{cc}
{\left[\frac{(0.73-0.1) \times L}{1.5-0.1}(b-0.1)+0.1 \times L\right]} & b=0.1, \ldots, 1.5 \\
{\left[\frac{(0.97-0.73) \times L}{2.7-1.5}(b-1.5)+0.73 \times L\right]} & b=1.6, \ldots, 2.7 \\
{\left[\frac{(1-0.97) \times L}{4.0-2.7}(b-2.7)+0.97 \times L\right]} & b=2.8, \ldots, 4.0 \\
L & b=4.1, \ldots, 16.0
\end{array}\right.
$$

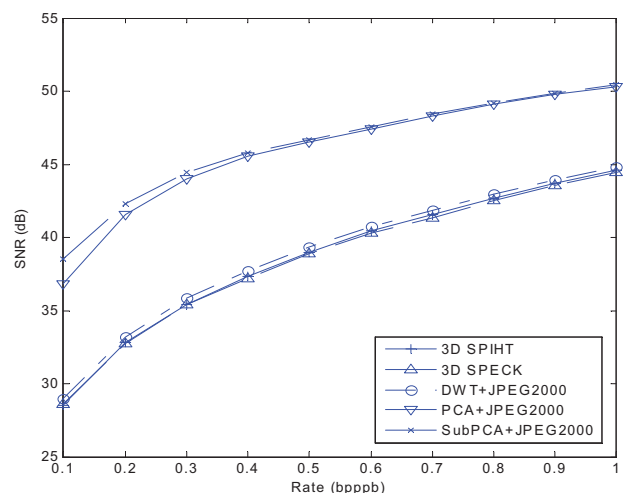

Fig. 1. Rate-distortion performance for AVIRIS Jasper Ridge data of size $512 \times 512$ with 224 bands.

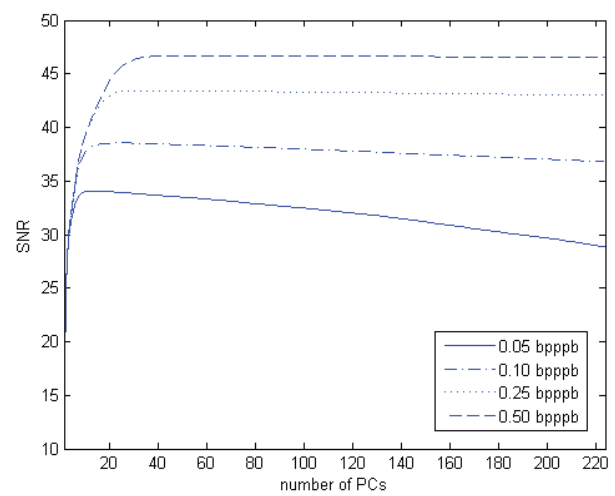

Fig. 2. The impact of the number of PCs maintained when using SubPCA+JPEG2000.

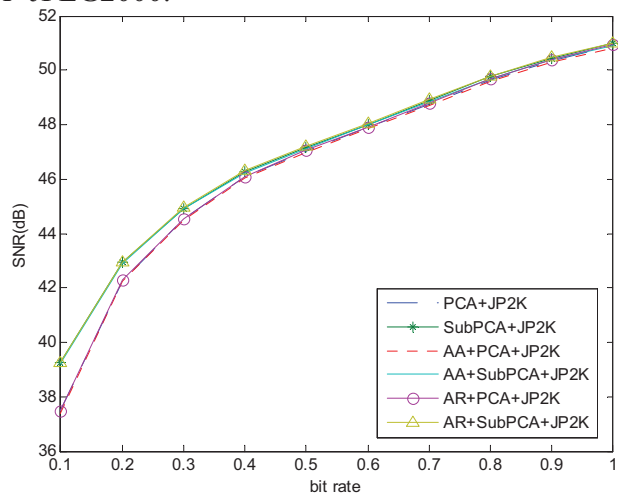

Fig. 3. The rate distortion curves from various algorithms for anomaly preservation.

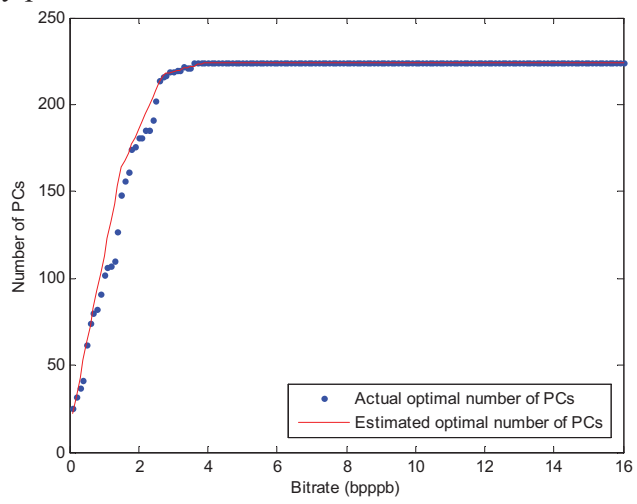

Fig. 4. Piecewise linear estimation of optimal number of PCs for SubPCA+JPEG2000. 
Here, $b$ represents bitrate in bit per pixel per band (bpppb), $L$ is the total number of bands, and $N_{P C}$ is the number of PCs to used for SubPCA+JPEG2000 for the bitrate $b$ to achieve the maximum SNR.

\section{Determination of Operational Bitrate}

After $N_{s}$ and $N_{a}$. are estimated with the procedure described in Sec. II-A, we set $N_{P C}$ to be equal to $N_{s}$ since we argue that all these signal components should be well maintained by the compression process. Then, based on (1), the corresponding bitrate $b=f^{-1}\left(N_{s}\right)$ is determined. We then adjust $b$ to accommodate the anomalous pixels that are transmitted uncompressed as overhead in the $\mathrm{AR}+\mathrm{SubPCA}+\mathrm{JPEG} 2000$ bitstream. The final bitrate, $b_{a c t}$, is slightly larger than $b$ :

$$
b_{a c t}=f^{-1}\left(N_{s}\right)+\frac{32 N_{a}}{N_{p}}
$$

where $N_{p}$ is the total number of pixels in the image. Here, it is assumed that anomalous pixels are represented using 32bit floating-point values. Although the spatial coordinates of anomalies need also be recorded in the $\mathrm{AR}+\mathrm{SubPCA}+\mathrm{JPEG} 2000$ bitstream, this overhead is negligible.

\section{EXPERIMENT}

We test our algorithm on two popular datasets: AVIRIS Indian Pine dataset (202 bands with $145 \times 145$ pixels) and ROSIS University of Pavia (103 bands with 610x340 pixels) dataset. $b_{a c t}$ for these datasets are 0.31 and 0.95 bpppb, and the corresponding numbers of PCs are 19 and 48, respectively. Figs. 1 and 2 show the classification maps from the support vector machine (SVM) classifier, where the one using the original data and the one using the $\mathrm{AR}+\mathrm{SubPCA}+\mathrm{JPEG} 2000$ reconstructed data are very similar. Table I lists the overall classification accuracy with different bitrates, where we can see that using $b_{a c t}$ and a small number of PCs, the classification accuracy can be similar to or even better than that using the original data. Note that for those using all the PCs (202 for Indian Pine, 103 for Pavia), the compression method is PCA+JPEG2000, so they cannot achieve perfect anomaly detection.

\section{CONCLUSION}

Currently existing lossy compression algorithms do not provide an approximately optimal bitrate that should be used to compress a hyperspectral image such that both the significant data information as well as the anomalies are preserved. Intuitively, only the significant PCs need be retained since they contain the majority of the information in the dataset; on the other hand, since anomalies tend to reside in minor components, they should be extracted and losslessly preserved before compression. Based on our previously developed AR+SubPCA+JPEG2000 compression technique, we propose an operational approach to determine the approximately optimal bitrate to be used to preserve both the majority of the information in the dataset as well as the anomalous pixels. The classification results using the reconstructed data after AR+SubPCA+JPEG2000 compression with this bitrate are comparable to those using the original data without compression, with $100 \%$ detection accuracy due to the fact that all the anomalies are intact.

\section{REFERENCES}

[1] J. E. Fowler and J. T. Rucker, "3D Wavelet-Based Compression of Hyperspectral Imagery," in Hyperspectral Data Exploitation: Theory and Applications, C.-I. Chang, Ed., John Wiley \& Sons, Inc., Hoboken, NJ, 2007.

[2] B. Penna, T. Tillo, E. Magli, and G. Olmo, "Progressive 3-D coding of hyperspectral images based on JPEG 2000," IEEE Geosciences and Remote Sensing Letters, vol. 3, no. 1, pp. 125-129, Jan. 2006.

[3] B. Penna, T. Tillo, E. Magli, and G. Olmo, "Transform coding techniques for lossy hyperspectral data compression," IEEE Trans. on Geosciences and Remote Sensing, vol. 45, no. 5, pp. 1408-1421, May 2007.

[4] Q. Du and J. E. Fowler, "Hyperspectral image compression using JPEG2000 and principal components analysis," IEEE Geoscience and Remote Sensing Letters, vol. 4, no. 2, pp. 201-205, April 2007.

[5] B.-J. Kim, Z. Xiong, and W. A. Pearlman, "Low bit-rate scalable video coding with 3D set partitioning in hierarchical trees (3D SPIHT)," IEEE Trans. on Circuits and Systems for Video Technology, vol. 10, pp. 1374-1387, 2000.

[6] X. Tang and W. A. Pearlman, "Scalable hyperspectral image coding," Proceedings on IEEE International Conference on Acoustics, Speech, and Signal Processing, vol. 2, pp. 401404, 2005.

[7] B. Penna, T. Tillo, E. Magli, and G. Olmo, "Hyperspectral image compression employing a model of anomalous pixels," IEEE Geosciences and Remote Sensing Letters, vol. 4, no. 4, pp. 664-668, Oct. 2007.

[8] Q. Du, W. Zhu, and J. E. Fowler, “Anomaly-based JPEG2000 compression of hyperspectal imagery," IEEE Geoscience and Remote Sensing Letters, vol. 5, no. 4, pp. 696-700, Oct. 2008.

[9] C. I. Chang and Q. Du, "Estimation of number of spectrally distinct signal sources in hyperspectral imagery," IEEE 
Transactions on Geoscience and Remote Sensing, vol. 42, no. 3, March 2004.

[10] J. M. Bioucas-Dias and J. M. P. Nascimento, "Hyperspectral subspace identification," IEEE Transactions on Geoscience and Remote Sensing, vol. 46, no. 8, pp. 2435-2445, Aug. 2008.

[11] N. Acito, M. Diani, and G. Corsini, "A new algorithm for robust estimation of the signal subspace in hyperspectral images in presence of rare signal components" IEEE
Transactions on Geoscience and Remote Sensing, vol. 47, no. 11, pp. 3844-3856, Nov. 2009.

[12] W. Zhu, PCA and JPEG2000-based Lossy Compression for Hyperspectral Imagery, Ph. D. Dissertation, Mississippi State University, May 2011.

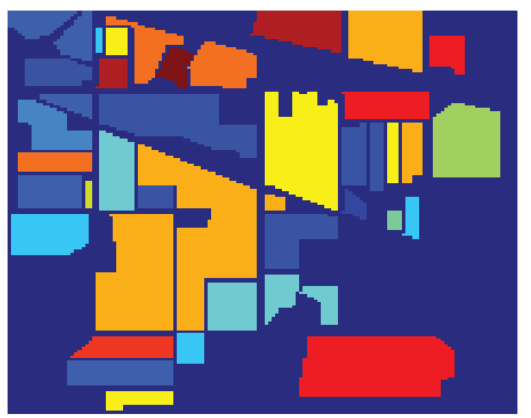

Ground Truth

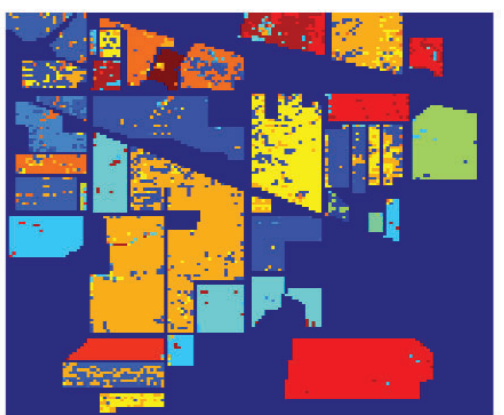

Original Data

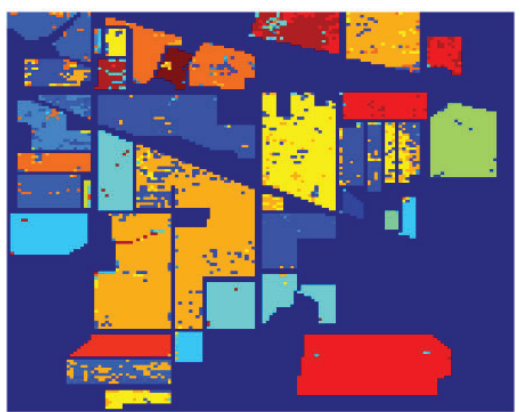

Reconstructed data (compressed by $b_{a c t}$ )

Fig. 1 The experiment using Indina Pine data

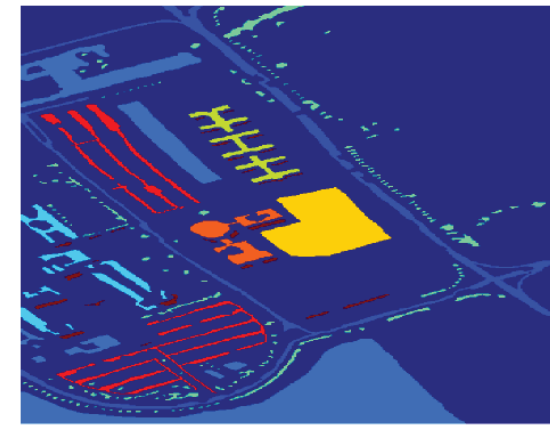

Ground Truth

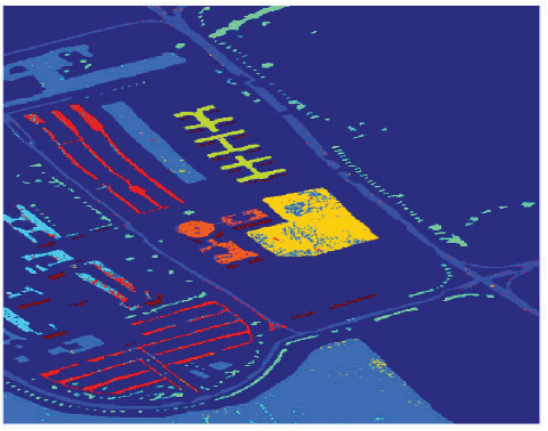

Original Data

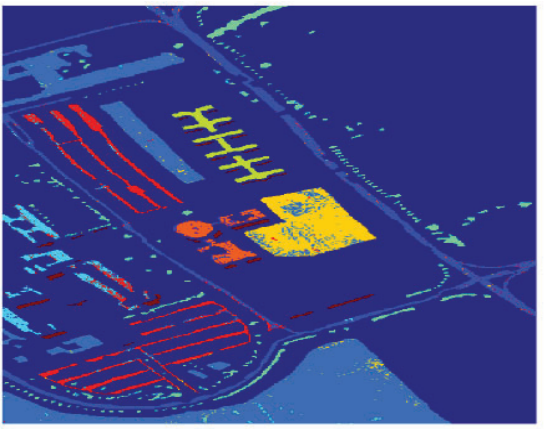

Reconstructed data (compressed by $b_{a c t}$ )

Fig. 2 The experiment using Pavia data

TABLE I

ClASSIFICATION ACCURACY USING THE ORIGINAL AND THE RECONSTRUCTED DATA

\begin{tabular}{|c|c|c|c|c|c|c|c|}
\hline Dataset & $\begin{array}{c}\text { Using } \\
\text { Original data }\end{array}$ & \multicolumn{6}{|c|}{$\begin{array}{l}\text { Using Reconstructed data } \\
\text { Classification Accuracy (with the corresponding bitrate, and the number of PCs) }\end{array}$} \\
\hline Indian Pine & 82.75 & $\begin{array}{c}87.15 \\
(0.31,19)\end{array}$ & $\begin{array}{c}86.43 \\
(0.1,10) \\
\\
87.38 \\
(0.35,204)\end{array}$ & $\begin{array}{c}87.13 \\
(0.3,19) \\
87.80 \\
(0.5,204)\end{array}$ & $\begin{array}{c}87.16 \\
(0.5,54) \\
87.21 \\
(0.7,204)\end{array}$ & $\begin{array}{c}86.31 \\
(0.7,90) \\
86.70 \\
(0.9,204)\end{array}$ & $\begin{array}{c}85.75 \\
(1.0,143) \\
86.41 \\
(1.0,204)\end{array}$ \\
\hline Pavia & 93.35 & $\begin{array}{c}93.22 \\
(0.95,48)\end{array}$ & $\begin{array}{c}93.19 \\
(0.1,10) \\
93.76 \\
(0.1,103) \\
\end{array}$ & $\begin{array}{c}93.86 \\
(0.3,19) \\
94.52 \\
(0.3,103) \\
\end{array}$ & $\begin{array}{c}93.38 \\
(0.5,28) \\
93.62 \\
(0.5,103) \\
\end{array}$ & $\begin{array}{c}93.14 \\
(0.7,37) \\
93.30 \\
(0.7,103) \\
\end{array}$ & $\begin{array}{c}92.98 \\
(1.0,51) \\
93.12 \\
(1.0,103) \\
\end{array}$ \\
\hline
\end{tabular}

